\title{
TEMPERATURAS DAS DIETAS DE PACIENTES SERVIDAS EM UM HOSPITAL
}

\section{TEMPERATURES OF PATIENT DIETS SERVED IN A HOSPITAL}

\author{
Camila Kelly de Sena Souza ${ }^{1}$ \\ Mônica Gloria Neumann Spinelli ${ }^{2}$ \\ Andrea Carvalheiro Guerra Matias ${ }^{2}$
}

Resumo: Para que o alimento possa cumprir seu papel de mantenedor do estado nutricional dos pacientes, é preciso considerar a composição nutricional, a qualidade microbiológica e os aspectos organolépticos da refeição. Nesse contexto, este estudo teve por objetivo avaliar a temperatura das preparações destinadas aos pacientes de um hospital particular em São Paulo. As refeições são acondicionadas em dois tipos de recipientes: pratos retornáveis e pratos térmicos descartáveis capazes de suportar reaquecimento em forno micro-ondas. Neste trabalho, foram analisadas as dietas oferecidas no almoço e jantar dos pacientes em sete refeições. A temperatura foi aferida na montagem, e antes da entrega ao paciente com um termômetro a laser. Todos os alimentos apresentaram temperaturas corretas ao final da cocção, porém apenas 5,4\% das preparações se encontravam em temperatura de segurança no momento da montagem das bandejas dos pacientes. Nas copas, 8,9\% das preparações se encontravam abaixo da temperatura correta, após o reaquecimento. Apesar do reaquecimento final possivelmente melhorar a aceitação da dieta, a qualidade microbiológica pode estar comprometida, uma vez que os alimentos foram mantidos em temperatura inadequada entre uma e duas horas. Torna-se necessário investimento em novos equipamentos no local de produção, para evitar perda da temperatura antes da montagem das bandejas com consequente aumento do tempo de exposição dessas preparações em temperaturas inadequadas, além de manutenção preventiva e corretiva dos equipamentos utilizados no local de distribuição, treinamento dos funcionários e monitoramento constante da temperatura durante todo processo produtivo.

Palavras-chave: dietas hospitalares; temperatura dos alimentos; qualidade dos alimentos.

Abstract: In order to make possible the food fulfill its role as the maintainer of nutritional status of patients, the nutritional composition, microbiological quality and organoleptic aspects of the meal must be consider. In this context, this paper presents a study that aimed to assess the temperature of patient preparations at a private hospital in São Paulo. Meals are packed in two types of containers: returnable and disposable thermal dishes capable of withstand microwave reheat. In this study, we analyzed the patient diets offered at lunch and dinner for seven meals. The temperature of the meal was measured in trays assembly and before delivery to the patient with a laser thermometer. All foods reached an adequate temperature at the end of cooking, but only $5.4 \%$ of preparations were in safety temperature at the time of mounting patient's trays. In galley kitchens, $8.9 \%$ of preparations were below the correct temperature after reheating. Despite the final rewarming possibly improve the acceptance of the diet, the microbiological quality can be compromised, since foods were kept in inadequate temperature between one and two hours. It is necessary to invest in new equipment in the food production site, to avoid loss of temperature before assembling the trays with consequent increase in the exposure time of these preparations at inappropriate temperatures, as well as preventive and corrective maintenance of the equipment used in place of distribution, employee training and constant temperature monitoring throughout the production process.

Keywords: hospital diets; food temperature; food quality.

\footnotetext{
${ }^{1}$ Graduanda em Nutrição - Universidade Presbiteriana Mackenzie - UPM, Brasil. E-mail: camila.ksena@gmail.com.

${ }^{2}$ Doutora em Saúde Pública (área de concentração - Nutrição) - Universidade de São Paulo - USP, Brasil. Docente do curso de Nutrição da UPM, Brasil. E-mail: spinelli@mackenzie.br; acgmatias@gmail.com.
} 


\section{INTRODUÇÂO}

A estrutura dos hospitais tem mudado ao longo do tempo, estreitando cada vez mais a ligação entre as partes do setor de saúde, a fim de que os serviços sejam otimizados para a promoção e proteção da saúde de modo geral. Para isso, os hospitais possuem equipe multiprofissional orientada em cuidados individuais, mas para atuação conjunta (WHO, 2014).

A postura das unidades hospitalares tem sido a de humanizar o serviço do cuidado com o paciente na atenção básica, nos atendimentos de urgência e emergência, na atenção especializada e na atenção hospitalar. Essa humanização do serviço tem como princípio básico o estímulo a processos comprometidos com a produção de saúde e com a produção de sujeitos, entre outros (BRASIL, 2003).

No papel que cabe ao serviço de nutrição, além de acompanhar o estado nutricional dos pacientes, os profissionais ficam também responsáveis pela produção e distribuição das refeições que devem ser adequadas individualmente para prevenção e tratamento de enfermidades. Todos os alimentos distribuídos/produzidos são de responsabilidade da Unidade de Alimentação e Nutrição (UAN) que é comandada por nutricionistas e técnicos em nutrição (PINHEIRO, 2010).

Em trabalho realizado por Sousa et al. (2011), foi destacado que a alimentação hospitalar é um serviço de grande complexidade, pois deve abranger e harmonizar as necessidades nutricionais dos pacientes com as responsabilidades e limitações econômico-administrativas e a programação da produção. Além disso, outro grande desafio é lidar com a percepção conotativa fortemente ligada à comida de hospital, que é comumente descrita como insossa, sem gosto, fria, servida cedo e sempre com um lembrete de proibição.

Para que o alimento possa cumprir, efetivamente, seu papel de restaurador e mantenedor do estado nutricional dos pacientes, é preciso levar em conta não somente a composição nutricional e a distribuição do conteúdo de macronutrientes, mas também é muito importante considerar a qualidade microbiológica e os aspectos organolépticos da refeição. Portanto, textura, aroma, cor, sabor e temperatura são fatores determinantes na qualidade da dieta ofertada aos pacientes de uma unidade hospitalar (SOUSA et al., 2011; PINHEIRO, 2010).

Em diversos estudos sobre aceitação da dieta hospitalar, fica evidente a importância da ingestão da dieta pelo paciente, pois, além de ser adequada qualitativamente para que as necessidades individuais sejam supridas, a alimentação exerce significado emocional relevante para a aceitação do tratamento e recuperação do paciente e está ligada a fatores sociais, culturais, étnicos e religiosos (DEMÁRIO; SOUSA; SALLES, 2010; COLOÇO; HOLANDA; PORTERO-MCLELLAN, 2009).

Coloço, Holanda e Portero-Mclellan (2009), em trabalho que avaliou o grau de satisfação da dieta oferecida pelo Programa Saúde da Família, não encontraram 
insatisfação relevante na unidade avaliada, mas ressaltaram que a estrutura física do local era favorável, além da disposição de carros térmicos que eram capazes de preservar a temperatura dos alimentos. Em seu levantamento bibliográfico encontraram um estudo de Yabuta, Cardoso e Isosaki (2006), no qual 70\% da amostra avaliada considerava a temperatura dos alimentos o aspecto mais relevante do serviço de alimentação; outro estudo, de Stanga et al. (2003) apontou, em 41,5\% da amostra, a temperatura como quesito de maior influência na aceitação da dieta.

Na maioria das vezes, a rotina hospitalar é adequada de forma mais conveniente para as equipes de saúde ao invés de satisfazer as necessidades pessoais dos indivíduos internados na unidade. A fim de aprimorar a relação entre as equipes e os pacientes, o Ministério da Saúde desenvolveu o Programa Nacional de Humanização da Assistência Hospitalar (PNHAH) (COLOÇO; HOLANDA; PORTERO-MCLELLAN, 2009).

Em se tratando da qualidade microbiológica, esta pode ser comprometida em diversas etapas da produção, que vai do recebimento da matéria-prima até a chegada da dieta aos leitos. Em todas as etapas da produção, existe o risco de contaminação e a veiculação das doenças transmitidas por alimentos (DTAs), risco esse diretamente proporcional à quantidade a ser produzida, ou seja, quanto maior a quantidade e variedade produzida, mais difícil o controle efetivo das etapas do preparo. A fim de garantir a segurança alimentar, existem legislações específicas que estabelecem normas técnicas para todas as etapas da produção e distribuição de alimentos (SOUSA; CAMPOS, 2003).

As normas técnicas que abrangem as boas práticas para manipulação de alimentos estão descritas em legislação específica para os serviços de alimentação através da RDC-216 (BRASIL, 2004). Dentre todos seus dizeres, o Manual de Boas Práticas (MBP) e os Procedimentos Operacionais Padronizados (POPs) descrevem os procedimentos a serem seguidos para que seja assegurada a qualidade dos alimentos (PINHEIRO, 2010).

A Portaria Estadual CVS-5 (BRASIL, 2013) e a Portaria Municipal $n^{\circ} 2619$ (BRASIL, 2011) também são instrumentos norteadores que padronizam todas as atividades dos diversos tipos de UANs.

A temperatura dos alimentos nas diversas etapas da produção é fator que interfere não só no aspecto biológico do alimento, mas também nas suas características organolépticas, uma vez que se não são seguidas as instruções adequadas, pode haver alterações de cor, cheiro, textura e, até mesmo, a perda do produto. Durante a produção, deve-se sempre considerar a relação tempo e temperatura para controlar a qualidade biológica e sensorial dos alimentos.

Em estudo realizado por Pinheiro (2010), que objetivou identificar possíveis pontos críticos de controle em todas as etapas da produção, foram encontradas 
inadequações de temperatura das preparações em tempo de espera (logo após a cocção); nas estufas, onde as cubas eram armazenadas e nos balcões e carros térmicos usados para distribuição.

Para enfatizar a importância das Boas Práticas de Manipulação (BPM), pode-se citar o estudo de Sousa e Campos (2003), que analisaram as condições higiênicosanitárias de uma dieta hospitalar e encontraram em $100 \%$ de suas amostras analisadas um número cem vezes maior que o permitido de coliformes termotolerantes, o que qualifica os alimentos impróprios para consumo. Outros estudos que encontraram não conformidades semelhantes, como medida para redução de riscos, os autores apontam, dentre outros, o controle da temperatura dos alimentos nas diversas etapas, principalmente durante o porcionamento e distribuição das refeições (PEDROSO et al., 1999; SALLES; GOULART, 1997).

Além disso, a aplicação de instrumentos que permitem avaliar a aceitação dos pacientes, não apenas no que diz respeito à alimentação, mas ao atendimento como um todo, se faz necessária, pois quanto mais as instituições conhecem seu público, mais fácil será suprir suas necessidades.

Segundo Troutner et al. (2012), o acompanhamento da temperatura dos alimentos desde o início da produção até o término do serviço e a identificação dos pontos em que ocorrem as alterações de temperatura podem auxiliar os administradores a melhorarem as operações.

Dessa forma, torna-se evidente a necessidade e importância do controle efetivo da temperatura dos alimentos produzidos e distribuídos aos pacientes, de forma que seja garantida a segurança microbiológica das refeições, bem como mantidas as características sensoriais de forma agradável, a fim de restabelecer o estado nutricional e o quadro geral do paciente. Nesse contexto este estudo teve por objetivo avaliar a temperatura das preparações destinadas aos pacientes de um hospital particular em São Paulo.

\section{METODOLOGIA}

Estudo de delineamento transversal aplicado em um hospital particular de grande porte, localizado na zona leste de São Paulo.

Neste trabalho, foram analisadas as dietas oferecidas no almoço e jantar dos pacientes. No horário do almoço, as dietas são compostas por arroz, feijão (só caldo, na branda), guarnição e prato principal; no jantar, compõem o prato: arroz, sopa, guarnição e prato principal.

As refeições são acondicionadas em dois tipos de recipientes: pratos térmicos moldados, com cavidade arredondada, fabricados com plástico acrinolitrila butadieno estireno ( $A B S$ ) de alta resistência com isolamento térmico na base e na tampa, com 
refil descartável, fabricado com poliestireno de alto impacto (PSAI), retornáveis e empilháveis; e pratos térmicos descartáveis, em PSAl, capazes de suportar reaquecimento em forno de micro-ondas.

Após o preparo, os alimentos que compõem as dietas, geralmente, são mantidos até o momento da distribuição em banho-maria. Essa é uma técnica de manutenção de temperatura em que os recipientes com alimentos ficam imersos em água com temperatura entre $80^{\circ} \mathrm{C}$ e $100^{\circ} \mathrm{C}$, segundo Sant'Ana (2012). Depois de servidos, os pratos são organizados em carros de transporte e levados até a copa do andar de destino. Lá, as refeições servidas nos recipientes descartáveis são reaquecidas, em micro-ondas, durante 3 (três) minutos. Ao término desse processo, os pratos são entregues nos quartos.

As copeiras e os andares acompanhados foram escolhidos de forma que a colheita de dados fosse realizada nos dois prédios que compõem o hospital, por quatro dias o almoço e três dias o jantar.

A temperatura dos alimentos foi aferida em dois momentos distintos:

Montagem: ao final da montagem de cada prato, antes de ser fechado;

Distribuição: entrega da dieta aos pacientes no andar de internação, após reaquecimento (no caso das embalagens descartáveis).

Para o envase das dietas, as copeiras vão até a área de montagem das dietas, que fica na cozinha do hospital, e cada uma fica responsável por montar os pratos de seu respectivo andar.

Ao término da montagem, cada copeira fica encarregada de levar seu carrinho para o andar e a distribuição é feita quase que imediatamente.

No almoço, a montagem ocorre entre $9 \mathrm{~h} 30$ e $10 \mathrm{~h}$, o reaquecimento na copa tem duração de, aproximadamente, 15 minutos (10h30 às 10h45) e as copeiras saem para entregar nos quartos às $11 \mathrm{~h}$.

No jantar, a montagem ocorre entre $15 \mathrm{~h}$ e $15 \mathrm{~h} 30$, o reaquecimento na copa tem duração de, aproximadamente, 15 minutos (16h10 às 16h25) e a entrega nos quartos às $16 \mathrm{~h} 30$.

A segunda temperatura foi aferida na copa, após a retirada dos pratos do aparelho de micro-ondas.

Para aferir a temperatura dos alimentos, foi usado um termômetro a laser da marca Emin modelo DT-8822 com alcance de $-50^{\circ} \mathrm{C}$ a $550^{\circ} \mathrm{C}$.

O trabalho seguiu todas as normas de ética e pesquisa e segurança no trabalho, tendo sido aprovado, protocolo CIEP no N008/03/13. 


\section{RESULTADOS E DISCUSSÃO}

Foram avaliados quatro dias no almoço e três no jantar e um total de 105 bandejas servidas a pacientes do hospital. As tabelas, a seguir (1, 2, 3 e 4), mostram a temperatura média de cada preparação, em cada dia da coleta de dados.

Tabela 1 - Temperatura média das preparações servidas no almoço em graus Celsius $\left({ }^{\circ} \mathrm{C}\right)$, na área de montagem, segundo tipo de embalagem. São Paulo, 2014.

\begin{tabular}{ccccccccc}
\hline & \multicolumn{2}{c}{ ARROZ } & \multicolumn{2}{c}{ FEIJÃO } & \multicolumn{2}{c}{ GUARNIÇÃO } & \multicolumn{2}{c}{$\begin{array}{c}\text { PRATO } \\
\text { PRINCIPAL }\end{array}$} \\
\cline { 2 - 9 } & ER & ED & ER & ED & ER & ED & ER & ED \\
\hline DIA 1 & 38,2 & 39,1 & 42,4 & 42,4 & 50,1 & 41,8 & 37,8 & 38,8 \\
DIA 2 & 50,7 & 43,9 & 48,1 & 46,2 & 48,9 & 61,2 & 42,3 & 45,6 \\
DIA 3 & 41,3 & 39,6 & 40,9 & 46,7 & 34,6 & 37,5 & 34,0 & 33,8 \\
DIA 4 & 35,7 & 40,6 & 51,5 & 52,0 & 52,5 & 47,5 & 29,2 & 30,5 \\
MEDIA & 41,5 & 40,8 & 45,7 & 46,8 & 46,5 & 47,0 & 35,8 & 37,2 \\
FINAL & & & & & & & & \\
DESVIO & 6,56 & 2,15 & 4,94 & 3,94 & 8,38 & 10,31 & 5,56 & 6,57 \\
PADRÃO & & & & & &
\end{tabular}

ER: embalagens plásticas reutilizáveis; ED: embalagens descartáveis.

Tabela 2. Temperatura média das preparações servidas no almoço em graus Celsius $\left({ }^{\circ} \mathrm{C}\right)$, na copa, segundo tipo de embalagem. São Paulo, 2014.

\begin{tabular}{lcccccccc}
\hline & \multicolumn{2}{c}{ ARROZ } & \multicolumn{2}{c}{ FEIJÃO } & \multicolumn{2}{c}{ GUARNIÇÃO } & \multicolumn{2}{c}{$\begin{array}{c}\text { PRATO } \\
\text { PRINCIPAL }\end{array}$} \\
\cline { 2 - 9 } & ER & ED & ER & ED & ER & ED & ER & ED \\
\hline DIA 1 & 36,8 & 51,7 & 35,7 & 47,1 & 42,0 & 46,4 & 35,6 & 42,7 \\
DIA 2 & 48,8 & 60,5 & 44,0 & 47,0 & 45,1 & 53,3 & 48,9 & 55,3 \\
DIA 3 & 49,7 & 51,7 & 47,3 & 51,8 & 52,3 & 55,9 & 49,6 & 47,3 \\
DIA 4 & 34,0 & 52,3 & 42,6 & 48,0 & 43,9 & 49,6 & 30,4 & 46,7 \\
MEDIA & 42,3 & 54,1 & 42,4 & 48,5 & 45,8 & 51,3 & 41,1 & 48,0 \\
FINAL & & & & & & & & \\
DESVIO & \multirow{2}{*}{$\mathbf{4} 08$} & 4,30 & 4,88 & 2,26 & 4,50 & 4,16 & 9,62 & 5,17 \\
PADRÃO & & & & &
\end{tabular}

ER: embalagens plásticas reutilizáveis; ED: embalagens descartáveis.

A temperatura é um fator de risco para a proliferação de micro-organismos. Para maior rapidez e facilidade de aferição da temperatura, foi utilizado um termômetro laser. Strasbourg et al. (2012) avaliaram diferenças de temperatura medidas com diferentes tipos de termômetro, constatando que o termômetro de espeto apresentou temperaturas superiores ao termômetro infravermelho laser- medida de superfície, com variações da ordem de 25 a $38 \%$. No entanto, nesse estudo foram avaliadas preparações distribuídas em serviços de alimentação coletiva, em utensílios de grande capacidade (gastronorm, cubas, rechaud), diferente deste caso, em que foram avaliados alimentos em pequenas porções. Deve-se observar que, na distribuição em grandes quantidades, a temperatura interna e externa apresentam maiores variações 
do que nos alimentos porcionados, em que a temperatura apresenta-se mais homogênea.

Tabela 3. Temperatura média das preparações servidas no jantar em graus Celsius $\left({ }^{\circ} \mathrm{C}\right)$, na área de montagem, segundo tipo de embalagem. São Paulo, 2014.

\begin{tabular}{ccccccccc}
\hline & \multicolumn{2}{c}{ ARROZ } & \multicolumn{2}{c}{ SOPA } & \multicolumn{2}{c}{ GUARNIÇÃO } & \multicolumn{2}{c}{$\begin{array}{c}\text { PRATO } \\
\text { PRINCIPAL }\end{array}$} \\
\cline { 2 - 9 } & ER & ED & ER & ED & ER & ED & ER & ED \\
\hline DIA 5 & 41,5 & 42,8 & 53,1 & 51,3 & 43,6 & 43,5 & 36,3 & 36,8 \\
DIA 6 & 40,4 & 37,2 & 49,0 & 53,1 & 43,4 & 47,4 & 40,4 & 47,4 \\
DIA 7 & 48,5 & 61,0 & 66,1 & 56,5 & 52,6 & 52,3 & 52,6 & 51,1 \\
MEDIA & 43,5 & 47,0 & 56,1 & 53,6 & 46,5 & 47,7 & 43,1 & 45,1 \\
FINAL & & & & & & & & \\
DESVIOO & 4,39 & 12,44 & 8,92 & 2,64 & 5,25 & 4,40 & 8,47 & 7,42 \\
PADRÃO & & & &
\end{tabular}

ER: embalagens plásticas reutilizáveis; ED: embalagens descartáveis

Tabela 4. Temperatura média das preparações servidas no jantar em graus Celsius $\left({ }^{\circ} \mathrm{C}\right)$, na copa, segundo tipo de embalagem. São Paulo, 2014.

\begin{tabular}{|c|c|c|c|c|c|c|c|c|}
\hline & \multicolumn{2}{|c|}{ ARROZ } & \multicolumn{2}{|c|}{ SOPA } & \multicolumn{2}{|c|}{ GUARNIÇÃO } & \multicolumn{2}{|c|}{$\begin{array}{c}\text { PRATO } \\
\text { PRINCIPAL }\end{array}$} \\
\hline & ER & ED & ER & ED & ER & ED & ER & ED \\
\hline DIA 5 & 38,8 & 51,9 & 50,1 & 48,5 & 40,9 & 46,1 & 35,9 & 46,1 \\
\hline DIA 6 & 38,7 & 51,5 & 42,7 & 51,1 & 38,9 & 46,7 & 37,7 & 50,7 \\
\hline DIA 7 & 49,3 & 62,1 & 54,0 & 61,1 & 47,4 & 62,2 & 51,9 & 66,0 \\
\hline $\begin{array}{l}\text { MEDIA } \\
\text { FINAL }\end{array}$ & 42,3 & 55,2 & 48,9 & 53,6 & 42,4 & 51,7 & 41,8 & 54,3 \\
\hline $\begin{array}{l}\text { DESVIO } \\
\text { PADRÃOO }\end{array}$ & 6,09 & 6,00 & 5,73 & 6,65 & 4,44 & 9,12 & 8,76 & 10,41 \\
\hline
\end{tabular}

ER: embalagens plásticas reutilizáveis; ED: embalagens descartáveis

Segundo a RDC-216 (ANVISA, 2004), após serem submetidos à cocção, os alimentos preparados devem ser mantidos em condições de tempo e de temperatura que não favoreçam a multiplicação microbiana. Para tal, os alimentos quentes devem ser mantidos acima de $60^{\circ} \mathrm{C}$, por até seis horas, sendo essas as condições adequadas para garantir a segurança do alimento.

A má conservação dos produtos, como, por exemplo, a carne, que por si só já é um fácil meio de proliferação, juntamente com as condições de temperatura e o tempo de exposição, torna-se uma forma fácil para uma intoxicação alimentar (SANSANA; BORTOLOZO, 2008).

Observa-se, nas Tabelas 1, 2, 3 e 4, que apenas 5,4\% das preparações se 
encontravam em temperatura de segurança aos consumidores, acima de $60^{\circ} \mathrm{C}$, no momento da montagem das bandejas dos pacientes. A temperatura dos alimentos na hora da finalização do preparo é controlada e se encontra adequada, porém as preparações são mantidas em banho-maria que, por serem insuficientes, não garantem que todos os alimentos fiquem em contato direto com a água quente. Algumas das inadequações cometidas durante a montagem das refeições durante 0 período do estudo foram principalmente a sobreposição de algumas cubas no banhomaria e a alocação de algumas cubas sobre balcão de inox sem qualquer tratamento térmico, sendo que as preparações mais frequentemente colocadas sob essas condições foram os pratos principais, justamente os alimentos que apresentaram menor temperatura durante a colheita de dados. Além da manutenção térmica inapropriada das cubas, o prato principal geralmente é uma preparação seca, como grelhados e assados, que apresenta maior dificuldade de manter a temperatura alta.

Nas copas, 8,9\% das preparações se encontravam abaixo da temperatura preconizada após o reaquecimento (Tabela 4). Este só é possível para as embalagens descartáveis e, apesar de haver um aumento de temperatura após este processo, ainda assim não é suficiente, pois são aquecidas quatro bandejas empilhadas por vez, com maior aquecimento nas bandejas inferior e superior. Apesar de o reaquecimento após a montagem possivelmente melhorar a aceitação da dieta, a qualidade microbiológica pode ficar comprometida, dependendo do tempo que os alimentos ficam mantidos em temperatura inadequada. A Portaria CVS-5/2013 não autoriza que alimentos com temperatura inferior a $60 \circ \mathrm{C}$ permaneçam mais de uma hora nessa condição.

Se comparados os dois tipos de embalagem (Tabelas 1, 2, 3 e 4), é possível notar que as descartáveis, por permitirem reaquecimento, ficam com uma temperatura pouco mais elevada. Dessa forma, a escolha do recipiente de servir é bastante importante, pois, além de contemplar a praticidade, a higienização e a apresentação dos alimentos, será mais um elemento no auxílio da manutenção da temperatura da dieta.

Antes da distribuição, apenas as refeições porcionadas em recipientes descartáveis, foram reaquecidas, pois as embalagens reutilizáveis não permitem tratamento em forno micro-ondas. Todos os pratos usados para controle, neste estudo, foram reaquecidos pelo mesmo período de tempo (três minutos), mas testes foram feitos com os pratos que foram excluídos do controle. Alguns foram reaquecidos por mais tempo (quatro e cinco minutos), o que alterou a estrutura física da embalagem, além de não apresentar diferença considerável na temperatura, quando comparados com os pratos aquecidos no tempo controle. Ao diminuir a quantidade de pratos colocados dentro do forno (geralmente colocados quatro pratos por vez) pelo mesmo tempo controle, a condição física da embalagem foi mantida e a temperatura dos alimentos teve um aumento de entre $5^{\circ} \mathrm{C}$ e $10^{\circ} \mathrm{C}$, dependendo do alimento, sendo que as preparações úmidas sempre apresentam temperatura superior à dos alimentos 
secos.

A temperatura das carnes, na área de montagem, variou de 29,2 a $52,6^{\circ} \mathrm{C}$ na área de montagem e nas copas, após o reaquecimento, de 30,4 a $66,0^{\circ} \mathrm{C}$. Na área de montagem, $100 \%$ dos pratos principais se encontravam em temperatura inadequada; e na copa, após o reaquecimento, apenas $7,1 \%$ atingiu a temperatura adequada. Devese ter mais preocupação com essas preparações, tendo em vista que esse tipo de alimento é rico em proteínas e possui alta atividade de água, quando in natura, que favorece o crescimento de micro-organismos. Por conta disso, esses alimentos costumam estar mais envolvidos em surtos de DTAs (SILVA JÚNIOR, 2013), quando não atingem a temperatura adequada durante cocção ou não se mantêm em temperatura adequada após a cocção.

Neste estudo, podem ser apontadas diferentes causas para não conformidades da temperatura do cardápio da cadeia quente (prato principal, guarnição e arroz), que estão atrelados à montagem das bandejas com o alimento já em temperatura inadequada, não acondicionamento das preparações, em utensílio que garanta a total conservação de temperatura, composição da preparação e aquecimento inadequado na copa.

Quanto à qualidade sensorial, a alimentação em temperatura inadequada, de um modo geral, favorece a pouca aceitação pelo paciente (TRANTER et al. 2009; WRIGHT; CONNELLY; CAPRA, 2006). Demário, Sousa; Salles (2010) também observaram que a refeição servida fria desagrada aos pacientes, embora estes reconheçam que os longos percursos da cozinha até o quarto promovam essa diminuição de temperatura.

\section{CONCLUSÃO}

Conclui-se que o controle do tempo e temperatura é fator importante na distribuição de refeições e devem ser monitorados constantemente. Baseados nesses resultados, os gestores das UANs hospitalares que utilizam sistema de distribuição de dietas centralizado, devem estar cientes dessas perdas de temperatura e promover meios para assegurar que essas sejam mantidas da melhor forma possível, além de garantir o menor tempo de distribuição possível. Portanto, torna-se necessário investimento em equipamentos adequados no local de produção, para evitar perda da temperatura antes da montagem das bandejas, com consequente perda de temperatura e aumento do tempo de exposição dessas preparações em temperaturas inadequadas, manutenção preventiva e corretiva dos equipamentos utilizados no local de distribuição, carros de transporte adequados, treinamento dos funcionários e monitoramento constante da temperatura, durante todo processo produtivo.

\section{REFERÊNCIAS}


ANVISA-Agência Nacional de Vigilância Sanitária. Regulamento Técnico de Boas Práticas de Manipulação para Serviços de Alimentação. Brasília: Avisa, 2004.

BRASIL. MINISTÉRIO DA SAÚDE. Humaniza SUS: política nacional de humanização. Brasília: Ministério da Saúde, 2003.

BRASIL. Centro de Vigilância Sanitária. Regulamento Técnico de Boas Práticas para Estabelecimentos Comerciais de Alimentos e Para Serviços de Alimentação. Portaria CVS n. 5, de 09 de abril de 2013. DOE, São Paulo, p. 32-35. 2013. Secretaria do Estado da Saúde. Disponível em:

<http://www.cvs.saude.sp.gov.br/up/PORTARIA\%20CVS-5_090413.pdf> Acesso em: 13 nov. 2014.

BRASIL. Secretaria Municipal de Saúde. Regulamento de Boas Práticas e de Controle de condições sanitárias e técnicas das atividades relacionadas à importação, exportação, extração, produção, manipulação, beneficiamento, acondicionamento, transporte, armazenamento, distribuição, embalagem e reembalagem, fracionamento, comercialização e uso de alimentos. Portaria n. 2619, 6 de dezembro de 2011. DOC, São Paulo, 2011. Secretaria Municipal da Saúde. Disponível em:

<http://www.prefeitura.sp.gov.br/cidade/secretarias/upload/chamadas/portaria_2619_1 323696514.pdf >. Acesso em: 13 de nov. 2014.

COLOÇO, R. B; HOLANDA, L. B; PORTERO-MCLELLAN, K. C. Determinantes do grau de satisfação de pacientes internados referente a refeições oferecidas em um hospital universitário. Rev. Ciênc. Med., Campinas-SP, v. 18, n. 3, p. 121-130, 2009.

DEMÁRIO, R. L; SOUSA, A. A; SALLES, R. K. Comida de hospital: percepções de pacientes em um hospital público com proposta de atendimento humanizado. Ciência \& Saúde Coletiva, v. 15, n. 1, p. 1275-1282, 2010.

PEDROSO, D. M. M. et al. Critical control points for meat balls and kibbe preparations in a hospital kitchen. Rev Microbiol, São Paulo, v. 30, n. 4, p. 347-355, 1999.

PINHEIRO, M. C. Análise da temperatura dos alimentos em um hospital público de Porto Alegre - RS: do preparo à distribuição ao paciente. UFRS, Porto Alegre, 2010.

SANSANA, D. C; BORTOLOZO, Q. E. Segurança Alimenta Domiciliar: conservação da carne mediante a aplicação do frio. In: SEMANA DE TECNOLOGIA EM ALIMENTOS, 6, 2008. Curitiba. Anais... Curitiba, v. 2, n. 39, 2008.

SANT'ANA, H. M. P. Planejamento físico-funcional de unidades de alimentação e nutrição. Rio de Janeiro: Editora Rubio, 2012.

SILVA Júnior, E. A. Manual de controle higiênico-sanitário em serviços de alimentação. São Paulo: Varela; 2013. 642 p.

SOUSA, A. A. et al. Aceitação de dietas em ambiente hospitalar. Revista de Nutrição, Campinas-SP, v. 24, n. 2, p. 287-294, 2011.

SOUSA, C. L.; CAMPOS, G.D. Condições higiênico-sanitárias de uma dieta hospitalar. Revista de Nutrição, Campinas - SP, v. 16, n. 1, p. 127-134, 2003.

STANGA, Z. et al. Hospital food: a survey of patients perceptions. Clin Nutr. v. 22, n. 3, p. 241-246, 2003. 
TRANTER, M. et al. Can patient-written comments help explain patient satisfaction with food quality? Journal of the American Dietetic Association, v. 109, n. 12, p. 2068-2072, 2009.

TROUTNER, M. et al. Analysis of Temperature of Patient Meals. Journal of Foodservice Management \& Education, v. 6, n. 2, p. 1-5, 2012.

WHO - WORLD HEALTH ORGANIZATION. Health topics: hospitals. 2014. Disponível em: <http://www.who.int/topics/hospitals/en/>. Acesso em: 13 nov. 2014.

WRIGHT, O. R.; CONNELLY, L. B.; CAPRA, S. Consumer evaluation of hospital foodservice quality: An empirical investigation. International Journal of Health Care Quality Assurance Incorporating Leadership in Health Services, v. 19, n. 2-3, p. 181-194, 2006.

YABUTA, C.Y.; CARDOSO, E.; ISOSAKI, M. Dieta hipossódica: aceitação por pacientes internados em hospital. Rev Bras Nutr Clin. v. 21, n. 1, p. 33-37, 2006. 